\section{Poste anatómico preformado: caso clínico}

Anatomic Preformed Post: Case Report

\section{Resumen}

En la actualidad los postes preformados se utilizan con mucha frecuencia, pero tienen la dificultad de no seguir la anatomía del conducto radicular. Obtener una forma más anatómica del conducto radicular reduciendo el espacio del cemento, contribuye a reducir la posibilidad de desalojo del mismo. En este artículo se detalla la confección de un poste anatómico preformado y las ventajas que representarían su uso clínico.

\author{
César Lamas Lara, ${ }^{1}$ Sergio Alvarado \\ Menacho, ${ }^{2}$ Rosa Pari Espinoza., ${ }^{3}$ \\ Cirujano Dentista, Docente del Área de \\ Operatoria Dental y Endodoncia de la \\ Facultad de Odontología de la UNMSM. \\ z Cirujano Dentista, Especialista en \\ Rehabilitación Oral, Profesor Asociado del \\ Área de Prótesis y Oclusión de la Facultad \\ de Odontologfa de la UNMSM \\ 3 Alumna del 5 to año de Odontología de la \\ UNMSM. \\ Correpondencia: \\ César Lamas Lara \\ Facultad de Odontología. UNMSM. Av. \\ Germán Amézaga s/n. Lima 1. Perú \\ Teléfono:999182287 \\ Correo electrónico: cesar2579@hotmail. \\ com
}

Palabras clave: Endodoncia, Poste Preformado, fibra de vidrio, cemento de resina.

Key words: Endodontic, Preformed post, Glass fiber, resin cement.

\section{Introducción}

Un poste ideal debe tener ciertas características para ser considerado como tal, como son la forma que debe ser similar al volumen dentario ausente, las propiedades mecánicas deben ser similares a las de la dentina, el desgaste estructural del diente debe ser el menor posible, debe ser resistente para soportar las fuerzas y el impacto masticatorio y su módulo de elasticidad debe ser lo más parecido a las estructuras histológicas que conforman el remanente dentario donde se va a trabajar dicho poste.

De lo anteriormente descrito como caracteristicas ideales de los postes, la que menos se cumple es la que el desgaste estructural del diente debe ser el menor posible, y esta es la razón que las endodoncias que se realizan en las piezas dentarias exigen un desgaste interno amplio para poder facilitar el procedimitiento clínico de la mejor manera; otra situación que se presenta es que muchas veces es requerido retirar postes previamente colocados por fra- caso de los mismos o por necesidades de re-tratamiento de las endodoncias que lo soportan. En este segundo caso la situación se vuelve crítica ya que es necesario retirar mayor estructura dentaria del conducto radicular con la finalidad de realizar el re-tratamiento endodóntico y por ende esto va en desmedro de la conservación de la estructura dentinaria intraradicular, ya que se crean preparaciones en forma de embudo las cuales obligan a realizar algún tipo de tratamiento que compense esa gran pérdida interna de estructura dentaria.

Colocar un "perno" debilita el diente en vez de hacerlo más resistente ya que su colocación requiere remoción adicional de dentina. ${ }^{1}$

Un muñón de resina en un poste intrarradicular rodeado por una corona de oro puede realizar la misma función y tener la misma resistencia que un munón colado de oro convencional. ${ }^{2}$

Los postes de fibra de carbono ofrecen un método resilente altamente retentivo y conservador para restaurar dientes endodóndicamente tratados. El procedimiento da como resultado la técnica del monobloque que viene a ser una sucesión de adhesión entre diente $_{\text {, cemento }}$, poste, reconstrucción coronaria y corona. Esta avanzada tecnología de adhesión asegura una gran resistencia a la fatiga y fractura, alta retención y comprobada estética. ${ }^{3}$

El primer antecedente de la colocación de postes preformados fue en 1983 , cuando Lovel propuso la utilización de fibras de carbono sumergidas en una matriz de naturaleza orgánica, pero de una manera muy artesanal. El gran aporte de los postes preformados de fibra se lo debemos a Duret que introdujo los postes de fibra de carbono reforzados con resina en $1988,4,5$

La retención de postes dentro del conducto radicular depende, en gran medida, de su diseño; longitud, forma, diámetro, superficie y en menor cuantía, del tipo de cemento utilizado. ${ }^{6}$

Los cementos que utilizamos funcionan bastante bien a espesores de película adecuados (el espesor de pe- 
lícula de los cementos de resina varía entre10-30 $\mu$ ), pero al tener postes (en este caso) con una forma preestablecida es imposible que este principio se cumpla, con la técnica que emplearemos tratamos de copiar a través de una resina compuesta la anatomía interna del conducto radicular y de esta manera reducir el espesor de película del agente cementante que vayamos a utilizar. ${ }^{78}$

Otro punto a tomar en consideración es el efecto de cuña que presentan los postes colados, ya que a mayor amplitud del conducto radicular mayor es el grosor del poste colado y por ende la probabilidad de fractura a nivel radicular si se usa este tipo de poste.

Los postes de fibra de vidrio presentan un módulo de elasticidad muy similar al de la dentina y esta característica nos brinda un factor favorable en nuestra rehabilitación. ${ }^{10}$

Este tipo de Postes Anatómicos fue planteado por primera vez por el Dr. Marco Ferrari afirmando que la presencia de un espesor exiguo de cemento determina una distribución más uniforme de las cargas oclusales y permite limitar la contracción de polimerización de la resina y el estrés determinado por ésta. ${ }^{5}$

\section{Caso clínico}

Paciente sexo masculino de 22 años de edad que acude a la Clínica de la Facultad de Odontología de la Universidad Nacional Mayor de San Marcos presentando restauraciones defectuosas en el sector anterior preocupado por su problema estético.

Al examinar el sector anterosuperior se puede apreciar tratamientos defectuosos a nivel de las piezas 1.2, 1.1, 2.1 y 2.2 .

Radiográficamente los retenedores intraradiculares presentan áreas de interfase con el conducto radicular, tratamientos de conductos en mal estadoy presencia de lesiones radiolúcidas a nivel periapical (figura 1).

\section{Diagnóstico}

Del estado de salud general: Paciente con buen estado de salud general, sin riesgo sistémico al tratamiento estomatológico.

Del estado de salud estomatológico: Paciente con presencia de periodontitis apical crónica en piezas 1.2, 2.1 y 2.2; y en la pieza 1.1 presenta abceso periapical crónico, además presenta tratamientos protésicos defectuosos.

\section{Plan de tratamiento}

Se procedió con el retiro de los postes colados de las piezas del sector anterosuperior; para posteriormente realizar los retratamientos respectivos.

De todas las piezas tratadas la pieza 1.1 presentaba mayor cantidad de problemas por resolver tanto desde el punto de vista endodóntico como protético. Se realizó el retratamiento de dicha pieza y se obturó con la técnica de impresión apical (figura 2).

Al apreciar la Radiografía de control se puede observar que las paredes del conducto radicular han quedado debilitadas por lo cual utilizar un Poste colado podría ser contraproducente por el efecto cuña que puede producir. Se decide realizar la colocación de un Poste Anatómico Preformado utilizando un poste de Fibra de Vidrio y Resina Compuesta ya que este tipo de postes tienen mejores propiedades mecánicas y de conducción de luz acorde con este caso.

\section{Procedimiento clínico de la conformación de un poste anatómico preformado}

Luego de haber realizado la desobturación de conducto radicular se realiza el aislamiento absoluto del campo operatorio, se coloca glicerina líquida dentro del conducto como medio aislante (figura 3 y 4). Se decidió utilizar un poste de fibra de vidrio por sus buenas propiedades estéticas y mecánicas; se le acondicionó con un sistema adhesivo de quinta generación para luego colocar sobre el poste resina de nanopartículas para copiar la anatomía interna del conducto radicular (figuras 5 y 6 ).

Una vez introducido el poste dentro del conducto radicular se fotocura 5 seg. Se retira el poste y se completa la polimerización fuera del conducto por 120 seg. (figuras 7 y 8 ). Posteriormente se realiza la confección del muñón con resina compuesta (figuras 9 y 10 ).

El poste anatomizado este es grabado con ácido ortofosfórico, lavado y silanizado. El conducto radicular es limpiado para eliminar los restos de glicerina que puedan quedar y es secado con conos de papel.

Se utilizó un cemento de resina dual autograbante y autoacondicionante, se asentó el poste, se retiraron los excesos y se fotopolimerizó el tiempo sugerido por el fabricante (figuras 11, 12, 13). Posteriormente se realizó la rehabilitación protésica de la pieza dentaria (figura 14).

\section{Discusión}

El conocimiento de las propiedades de los diferentes tipos de postes con los que contamos actualmente juega un papel primordial en el resultado de nuestra rehabilitación.

La decisión de colocar un poste preformado de fibra de vidrio no solo es necesario por las mejoras en las propiedades ópticas en relación a los postes colados sino también a sus mejores propiedades mecánicas. ${ }^{11,12}$

En algunos casos el uso de los postes de fibra de vidrio como material de elección por sus buenos resultados clínicos son defendidos por varios autores ya que superan ampliamente aspectos de procedimientos clínicos. ${ }^{13}$, 14 y 15

\section{Conclusiones}

El uso de postes anatómicos preformados asegura un espesor de pelicula del cemento más adecuado y de esta manera obtener mayor seguridad en nuestra restauración.

Es necesario una adecuada comunicación endodoncista rehabilitador para poder realizar la mejor elección del tipo de retenedor intraradicular que se va a colocar y de esta manera asegurarnos que nuestra restauración cumpla a cabalidad su propósito.

El avance de la odontología hace que los postes preformados sean cada día más de uso común en nuestra práctica odontológica y debemos de estar preparados en conocimientos teóricos y clínicos sobre el uso de los nuevos materiales en odontología restauradora.

\section{Referencias bibliográficas}

1. Sorensen J., Martinoff J. Intracoronal reinforcement and coronal converage. J. prost. Dent.1984; 1:780.

2. Ake Linde L. Uso de composites en combinación con un poste intrarradicular como muñón en una pieza tratada endodónticamente. Aspectos clínicos de la técnica, Quintessence (ed. Esp). 1995; 8(3): 10-16

3. Freedman G. Los postes de fibra de carbón. Rehabilitación postendodóntica adhesiva, Journal de Clínica en Odontologia. 1997; 3: 19-26.

4. Shillingburg HT, Hobo S, Whitsett L, Jacobi R, Brackett $S$. Preparación para dientes extensamente dañados. En: 
Fundamentos Esenciales en Prótesis Fija. 3 Ed. Madrid: Quintessence books; 2000:194-206

5. Ferrari M, Scotti R. Postes Anatómicos En: Postes de Fibra, Características y Aplicaciones Clínicas. Roma: Masson; 2002: 91-96

6. Zamorano P y col. Microestructutura de la zona de adhesion en conductos tratados endodónticamente. Revista dental de Chile, 2005; 96 (2): 3-6

7. Ferrari $\mathrm{M}$ y col. Bonding to root canal: Structural characteristics of the substrate, Am J Dent, $2000 ; 13: 255-60$.

8. Bonfante E y col, SEM observation of the bond integrity of fiber-reinforced composite posts cemented into root canals, Dental Materials 2008; 24 , 483-491

9. Assif D, Gorfil C. Biomechanical considerations in restoring endodontically treated teeth. J Prosthet Dent. 1994; 71(6):565- 7.

10. Chawla KK. Carbon Fiber Composites. En: Composite materials: science and engineering. $2 \mathrm{Ed}$. New York: Springer; 1998: $252-270$

11. Torbjörner A, Fransson B. A literatura review on the prosthetic treatment of structurally compromised teeth. Int I Prosthodont 2004;17:369-76.

12. Lippo L, Tanner J, Le Bell AM, Narva K, Vellittu P. Flexural properties of reinforced root canal post. Academy of Dental Materials 2004;20:29-36.
13. Bertoldi A. Nuevos enfoques en la reconstrucción coronaria del diente endodonciado. Rev Asoc Odont Argent 2002;4(90):157-62.

14. Monticelli F, Grandini S, Goracci C, Ferrari M. Comportamiento clínico de los pernos de fibra traslúcida: estudio prospectivo de 2 años. Revista internacional de prótesis estomatológica $2004 ; 6(4): 321-4$

15. Mannoci F, Qualtrough AJ, Worthington HV, Watson TF, Pitt Ford TR. Randomized clinical comparison of endodontically treated teethrestored with amalgam or with fiber post and resin composite: five year results. Oper Dent 2005:30(1):9-15.

Fecha de recepción: 13 abril 2009

Fecha de aceptación: 10 julio 2009

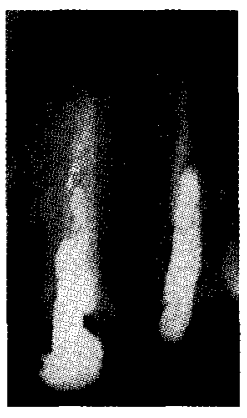

Fig. 1. Radiografía Inicial Pieza 2.1

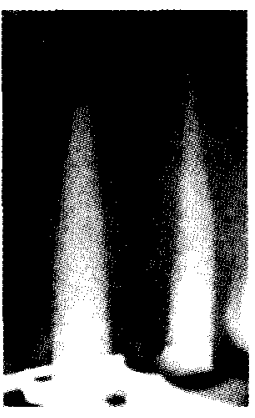

Fig. 2. Radiografía de Obturación

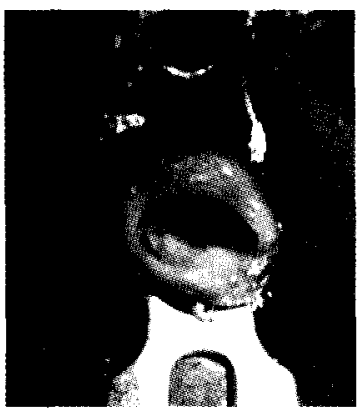

Fig. 3. Aislamiento Absoluto del campo operatorio.

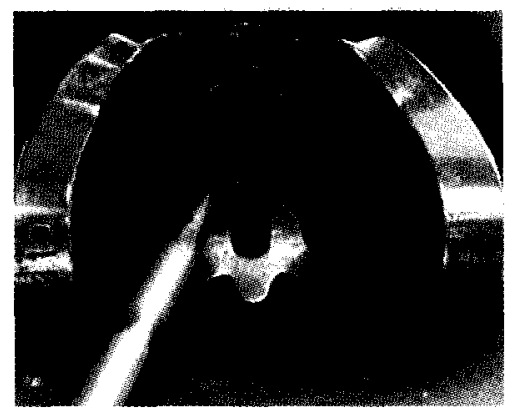

Fig. 4. Colocación de glicerina líquida como medio de aislante.

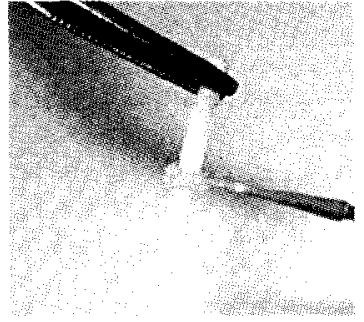

Fig. 5. Acondicionamiento del poste.

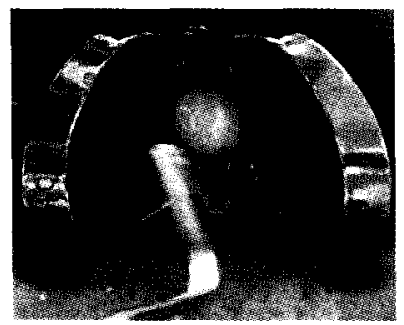

Fig. 9. Confección del Muñón.

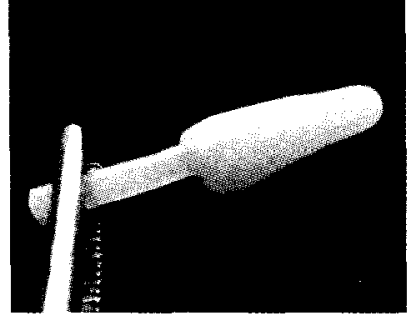

Fig. 6. Colocación de resina compuesta sobre el poste de fibra de vidrio para poder copiar la anatomía interna del conducto radicular.

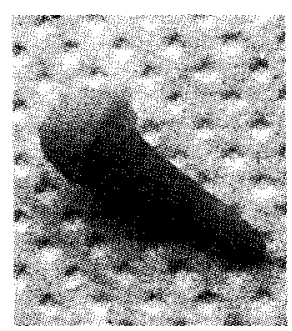

Fig. 10. Poste anatómico terminado.

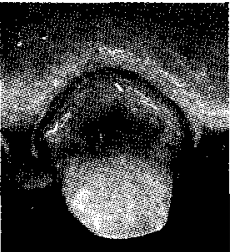

Fig. 13. Poste cementado.

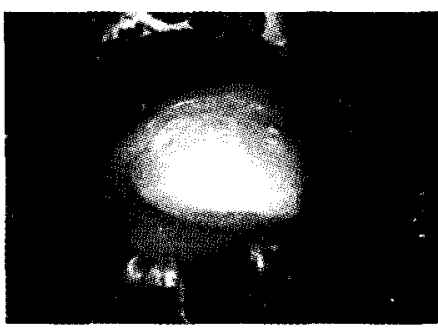

Fig. 11. Cementación del poste.

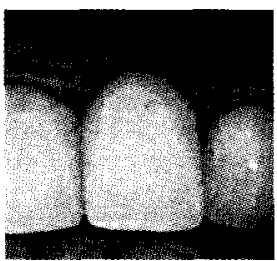

Fig. 14. Pieza con rehabilitación protésica terminada.

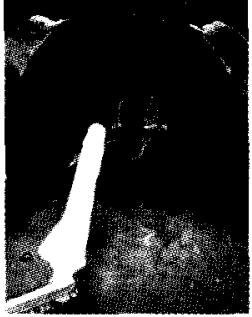

Fig. 8. Retiro de poste para completar la polimerización por 120 seg.

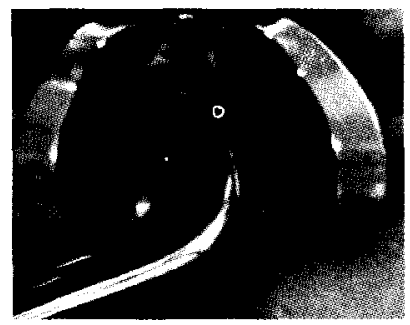

Fig. 12. Fotopolimerización. 